\title{
Outcome of Corona Virus Disease (COVID-19) in Egyptian Cohort of Long-Term Liver Transplant Recipients: Single Center Experience
}

\author{
Anwar Ismail ${ }^{1,2}$ (D) *, Said Mohamed ${ }^{1,2}$, Essam Mahmoud ${ }^{1,2}$, Hosny Karim $^{2,3}$, Doaa Mansour ${ }^{2,3}$, \\ Zayed Nagla ${ }^{2,4}$, Akram El- Adwy ${ }^{1,2}$, ElAmir Mona ${ }^{2,5}$, Mogawer Sherif ${ }^{2,5}$, Elshazly Mostafa ${ }^{2,3}$ \\ and Nabil Ahmed ${ }^{2,3}$
}

${ }^{1}$ Department of Endemic Medicine \& Hepatogastroenterology, Kasr Al Aiy Faculty of Medicine, Cairo University, Cairo, Egypt

${ }^{2}$ Liver Transplantation unit, Kasr Al Aiy Faculty of Medicine, Cairo University, Cairo, Egypt

${ }^{3}$ Department of General Surgery, Kasr Al Aiy Faculty of Medicine, Cairo University, Cairo, Egypt

${ }^{4}$ Anesthesia \& surgical ICU, Kasr Al Ainy Faculty of Medicine, Cairo University, Egypt

${ }^{5}$ Department of Internal Medicine, Kasr Al Aiy Faculty of Medicine, Cairo University, Cairo, Egypt

\begin{abstract}
Background: Our knowledge and experience about further management and follow up of patients following COVID-19 infection remains unknown.

Material and methods: We are documenting our preliminary experience from a single transplantation center, Manial specialized hospital. And the follow up records of patients who were diagnosed to have severe acute respiratory syndrome coronavirus 2 (SARS-CoV-2) From March 2020 to July 2020 were reviewed.

Results: Among 21 cases of COVID-19 infection in our center, median age at diagnosis was $40 \pm 12.8$ years, and the median interval since transplantation was 4.75 years. Fever (100\%) and radiographic abnormalities in form of unilateral or bilateral/ multifocal consolidations (80\%) were the most common presentations. Macrolide's antibiotics were used in $75 \%$ of patient.95\% was improved and $4.6 \%$ showed mortality, reduction or stoppage of current immunosuppressives were only in $19 \%$.

Conclusion: Management of severe acute respiratory syndrome following COVID-19 infection after solid organ transplantation is challenging with unpredicted outcome and need specialized care in this group of population with shared experience among Liver transplant experts with tailored management plan for each case.
\end{abstract}

Keywords

Liver transplantation, SARS-CoV-2, Consolidation, Pandemic

\section{Introduction}

Despite the world-wide COVID-19 pandemic, published data about liver transplantation and its fate still unclear [1].

Since January 2020, the novel COVID-19 disease resulted in a global pandemic making decisions about liver transplantation and its fate uncertain [2].

In our liver transplant (LT) center, seven cases were diagnosed to have COVID-19 based on epidemiological history of exposure, clinical manifestation, chest imaging and laboratory test.

The first reported case in post-transplant COVID-19 era was in a 52-year-old Chinese kidney transplant (KT) recipient,

\begin{abstract}
*Corresponding author: Ismail Anwar, MD Lecturer of Endemic Hepatology and Gastroenterology, Kasr Al Aiy Faculty of Medicine, Cairo University, Egypt, Tel: 002-01003342126

Accepted: August 25, 2021

Published online: August 27, 2021

Citation: Ismail A, Mohamed S, Mahmoud E, et al. (2021) Outcome of Corona Virus Disease (COVID-19) in Egyptian Cohort of Long-Term Liver Transplant Recipients: Single Center Experience. J Transplant Surg 4(2):80-86
\end{abstract}


Citation: Ismail A, Mohamed S, Mahmoud E, et al. (2021) Outcome of Corona Virus Disease (COVID-19) in Egyptian Cohort of Long-Term Liver Transplant Recipients: Single Center Experience. J Transplant Surg 4(2):80-86

with favorable outcome response following stoppage of the immunosuppression and low-dose methylprednisolone [3]. A second report was from Spain; however no information on outcome after diagnosis was available [4].

Patients post- liver transplantation is at a higher risk for developing septic shock following COVID-19 acute respiratory distress syndrome [5]. However other hypothesis may believe that the long-term post-transplant immunosuppression may interfere to some extent with the cytokine storm that leads to multiorgan failure and is responsible for most of SARS-CoV-2 attributable deaths [6].

The clinical course of COVID-19 in immunosuppressed transplant recipients may differ from that in nonimmunosuppressed patients. Indeed, while hepatocellular injury, as characterized by elevated serum aminotransferases, appears to be relatively less prevalent, acute kidney injury is more common in transplant recipients with COVID-19, possibly due to the use of calcineurin inhibitors [7].

\section{Patients, Methods and Outcome}

\section{Patients population}

Adults (age > 20 years) with post- liver transplantation recipients cared under our Manial specialized liver transplantation center. Data were retrospectively collected and analyzed with laboratory-confirmed novel SARS-COVID-19 infection in a time period from March 2020 to August 2020.

Data was obtained manually from our patient's medical records. We also directly communicated with the recipient and their family for simple questionnaire to ensure their safety. Only patients who met the diagnostic criteria of the new Coronavirus Pneumonia were included on the basis of epidemiological history of exposure, clinical manifestation, chest imaging and laboratory test of SARS-CoV-2 from the respiratory specimens by real-time reverse transcriptase polymerase chain reaction (RT-PCR) [8].

\section{Laboratory workup}

COVID-19 RNA testing of nasopharyngeal swab samples was performed using a real-time reverse transcriptasepolymerase chain reaction (rRT-PCR) assay based on a US Centers for Disease Control and Prevention assay approved by U.S. Food and Drug Administration Emergency Use Authorization (EUA) [9].

\section{Case series}

Out of 255 cases of liver transplant in our center, 21 (8.23\%) recipients had COVID-19 infection, Recipients were classified in to 17 (80.5\%) confirmed, 4 (19.04\%) Suspected and 19 (90.4\%) long term survivors with post-transplant period more than 12 months and only $2(9.5 \%)$ recently transplanted as shown in: Table 1, Table 2, Table 3 and Table 4.

\section{Demographic data and comorbidities}

The median age was 52.5 (age range from 47 to 68). Most of cases were men 18 (85\%). The median time from transplantation was 4.75 years (time range since transplantation ranging from 6 months to 9 years). Only one case had chronic rejection (4.7\%) and two cases had history of recent biliary stricture (9.5\%), rest, $90 \%$ had stable graft functions.

None of the cases were smokers, only one case is hypertensive, and two cases were diabetic with good control on insulin therapy.

None of them were taking an angiotensin-converting enzyme inhibitor or angiotensin receptor blocker, hydroxycholoroquine were taken by 2 (9.5\%). And all of them had BMI less than or equal $29 \mathrm{~kg} / \mathrm{m}^{2}$.

\section{Clinical presentation}

No cases have recent travel or contact with confirmed cases. However, all cases $(n=21)$ had the classical respiratory symptoms, common symptoms were fever (100\%), cough (100\%), dyspnea (90.4\%), myalgias (100\%), and fatigue (100\%). Less common symptoms were diarrhea $(14 \%)$ and anosmia/dysgeusia (9.6\%).

9 cases $(42.6 \%)$ required hospitalization and only a single case $(4.7 \%)$ had severe respiratory symptoms with severe hypoxia on room air $\left(\mathrm{SO}_{2}=88 \%\right.$ on room air and tachypnea $=$ 33) necessitating mechanical ventilation.

According to WHO assessment scale (this scale reflects a range from uninfected to dead, where 0 is "no clinical or virological evidence of infection", 1 is "no limitation of activities", 2 is "limitation of activities", 3 is "hospitalized, no oxygen therapy", 4 is "oxygen by mask or nasal prongs", 5 is "non-invasive ventilation or high-flow oxygen", 6 is "intubation and mechanical ventilation", 7 is "ventilation + additional organ support - pressors, RRT (renal replacement therapy), ECMO (extracorporeal membrane oxygenation)", and 8 is "death") [10].



Figure 1: Showing bilateral GCO with subpleural bands from one of our cases (CORADS 5, HIGHLY LIKELY COVID-19) 
Citation: Ismail A, Mohamed S, Mahmoud E, et al. (2021) Outcome of Corona Virus Disease (COVID-19) in Egyptian Cohort of Long-Term Liver Transplant Recipients: Single Center Experience. J Transplant Surg 4(2):80-86

Table 1: Case series of seven living donor liver transplant recipients with COVID-19 infection.

\begin{tabular}{|c|c|c|c|c|c|c|c|}
\hline & Case 1 & Case 2 & Case 3 & Case 4 & Case 5 & Case 6 & Case 7 \\
\hline Age (y)/sex & $52 /$ male & $50 /$ male & $49 /$ male & 51/male & $63 / \mathrm{male}$ & $51 /$ male & $55 /$ male \\
\hline BMI & $28 \mathrm{~kg} / \mathrm{m}^{2}$ & $27 \mathrm{~kg} / \mathrm{m}^{2}$ & $23 \mathrm{~kg} / \mathrm{m}^{2}$ & $26 \mathrm{~kg} / \mathrm{m}^{2}$ & $27 \mathrm{~kg} / \mathrm{m}^{2}$ & $25 \mathrm{~kg} / \mathrm{m}^{2}$ & $22 \mathrm{~kg} / \mathrm{m}^{2}$ \\
\hline $\begin{array}{l}\text { Duration post } \\
\text { LTx By months }\end{array}$ & 99 & 71 & 8 & 92 & 96 & 100 & 86 \\
\hline IS drugs & Tacrolimus & $\begin{array}{l}\text { Tacrolimus/ } \\
\text { MMF }\end{array}$ & Tacrolimus & Tacrolimus & Tacrolimus & Tacrolimus & Tacrolimus \\
\hline Fever & Yes & Yes & Yes & Yes & Yes & Yes & Yes \\
\hline $\begin{array}{l}\text { Recent travel or } \\
\text { COVID contact }\end{array}$ & No & No & No & No & No & No & No \\
\hline Diarrhea & No & NO & NO & NO & No & No & No \\
\hline $\begin{array}{l}\text { Duration of } \\
\text { symptoms }\end{array}$ & 14 days & 14 days & 15 days & 25 days & 12 days & 13 days & 10 days \\
\hline \multirow[t]{2}{*}{$\begin{array}{l}\text { Other } \\
\text { symptoms }\end{array}$} & $\begin{array}{l}\text { Myalgia and } \\
\text { dyspnea }\end{array}$ & $\begin{array}{l}\text { Myalgia, } \\
\text { dyspnea and } \\
\text { anosmia }\end{array}$ & $\begin{array}{l}\text { Myalgia, cough, } \\
\text { dyspnea }\end{array}$ & $\begin{array}{l}\text { Myalgia, cough } \\
\text { and dyspnea }\end{array}$ & $\begin{array}{l}\text { Cough and } \\
\text { Dyspnea }\end{array}$ & $\begin{array}{l}\text { Bony aches and } \\
\text { Myalgia }\end{array}$ & $\begin{array}{l}\text { Dyspnea, cough, } \\
\text { loss of taste }\end{array}$ \\
\hline & Case 8 & Case 9 & Case 10 & Case 11 & Case 12 & Case 13 & Case 14 \\
\hline Age (y)/sex & 58/male & 56/male & $47 /$ male & 56/male & $68 /$ male & 55/male & 54/male \\
\hline BMI & $25 \mathrm{~kg} / \mathrm{m}^{2}$ & $28 \mathrm{~kg} / \mathrm{m}^{2}$ & $23 \mathrm{~kg} / \mathrm{m}^{2}$ & $28 \mathrm{~kg} / \mathrm{m}^{2}$ & $27.5 \mathrm{~kg} / \mathrm{m}^{2}$ & $25 \mathrm{~kg} / \mathrm{m}^{2}$ & $24 \mathrm{~kg} / \mathrm{m}^{2}$ \\
\hline $\begin{array}{l}\text { Duration post LTX } \\
\text { By months }\end{array}$ & 108 & 72 & 6 & 96 & 96 & 108 & 72 \\
\hline IS drugs & $\begin{array}{l}\text { Tacrolimus/ } \\
\text { MMF }\end{array}$ & $\begin{array}{l}\text { Tacrolimus/ } \\
\text { MMF }\end{array}$ & $\begin{array}{l}\text { Tacrolimus/ } \\
\text { MMF }\end{array}$ & $\begin{array}{l}\text { Tacrolimus/ } \\
\text { Everolimus/MMF }\end{array}$ & Tacrolimus & Tacrolimus & Tacrolimus \\
\hline Fever & Yes & Yes & Yes & Yes & Yes & Yes & Yes \\
\hline $\begin{array}{l}\text { Recent travel or } \\
\text { COVID contact }\end{array}$ & No & No & No & No & No & No & No \\
\hline Diarrhea & No & NO & NO & YES & No & No & No \\
\hline $\begin{array}{l}\text { Duration of } \\
\text { symptoms }\end{array}$ & 14 days & 14 days & 10 days & 21 days & 10 days & 14 days & 7 days \\
\hline \multirow[t]{2}{*}{ Other symptoms } & $\begin{array}{l}\text { Myalgia and } \\
\text { dyspnea }\end{array}$ & $\begin{array}{l}\text { Myalgia, dyspnea } \\
\text { and anosmia }\end{array}$ & $\begin{array}{l}\text { Myalgia, cough, } \\
\text { dyspnea }\end{array}$ & $\begin{array}{l}\text { Myalgia, cough } \\
\text { and dyspnea }\end{array}$ & $\begin{array}{l}\text { Cough and } \\
\text { Dyspnea }\end{array}$ & $\begin{array}{l}\text { Bony aches and } \\
\text { Myalgia }\end{array}$ & Asymptomatic \\
\hline & Case 15 & Case 16 & Case 17 & Case 18 & Case 19 & Case 20 & Case 21 \\
\hline Age $(y) /$ sex & 51/female & $52 /$ male & 49/female & 47/female & $65 /$ female & 53/male & $49 /$ male \\
\hline BMI & $23 \mathrm{Kg} / \mathrm{m}^{2}$ & $25 \mathrm{~kg} / \mathrm{m}^{2}$ & $21 \mathrm{~kg} / \mathrm{m}^{2}$ & $28 \mathrm{~kg} / \mathrm{m}^{2}$ & $22 \mathrm{~kg} / \mathrm{m}^{2}$ & $27 \mathrm{Kgm} / \mathrm{m}^{2}$ & $24 \mathrm{Kg} / \mathrm{m}^{2}$ \\
\hline $\begin{array}{l}\text { Duration post LTX } \\
\text { By months }\end{array}$ & 98 & 108 & 66 & 72 & 98 & 108 & 58 \\
\hline IS drugs & Tacrolimus & Tacrolimus/ MMF & Tacrolimu & $\begin{array}{l}\text { Tacrolimus/ } \\
\text { Everolimus }\end{array}$ & Tacrolimus & Tacrolimus & Tacrolimus \\
\hline Fever & Yes & Yes & Yes & Yes & Yes & Yes & Yes \\
\hline $\begin{array}{l}\text { Recent travel or } \\
\text { COVID } \\
\text { contact }\end{array}$ & No & No & No & No & No & No & No \\
\hline Diarrhea & No & NO & NO & YES & No & No & No \\
\hline $\begin{array}{l}\text { Duration of } \\
\text { symptoms }\end{array}$ & 14 days & 14 days & 14 days & 21 days & 14 days & 14 days & 21 days \\
\hline Other symptoms & $\begin{array}{l}\text { Myalgia and } \\
\text { dyspnea }\end{array}$ & $\begin{array}{l}\text { Myalgia, dyspnea } \\
\text { and anosmia }\end{array}$ & $\begin{array}{l}\text { Myalgia, cough, } \\
\text { dyspnea }\end{array}$ & $\begin{array}{l}\text { Myalgia, cough } \\
\text { and dyspnea }\end{array}$ & $\begin{array}{l}\text { Cough and } \\
\text { Dyspnea }\end{array}$ & $\begin{array}{l}\text { Bony aches and } \\
\text { Myalgia }\end{array}$ & $\begin{array}{l}\text { Cough and } \\
\text { Dyspnea }\end{array}$ \\
\hline
\end{tabular}

$6(28 \%)$ were WHO scale $1,5(23 \%)$ were WHO scale 2,7 (33.3\%) were WHO scale 3, 2 (9.5\%) were WHO scale 4 and only $1(4.7 \%)$ were WHO scale 7.

\section{Laboratory studies}

All patients had lymphopenia (100\%), and 8 patients had elevated creatinine (38\%), all of the cases had elevated CRP and ferritin level Serum bilirubin was markedly elevated up to $33 \mathrm{mg} / \mathrm{dl}$ in one case (4.7\%). All patients were tested for nasopharyngeal COVID-RNA testing and were tested as positive except four cases were tested negative but with typical clinical and imaging findings.

\section{Imaging findings}

CT chest (Figure 1) was performed to all cases and most cases showed bilateral ground glass opacities (GGO) and consolidations (CORAD classification was 5) [11].

\section{Management plan, complications and outcome}

In line with clinical practice guidelines proposed by the Egyptian Ministry of Health and local protocols, Macrolide's antibiotics (Azithromycin $250 \mathrm{mg}$ twice daily orally for 3 days) together with vitamin $C$ and Zinc supplements for 10 days were prescribed to patients after written or oral informed 
Citation: Ismail A, Mohamed S, Mahmoud E, et al. (2021) Outcome of Corona Virus Disease (COVID-19) in Egyptian Cohort of Long-Term Liver Transplant Recipients: Single Center Experience. J Transplant Surg 4(2):80-86

Table 2: Showing WHO assessment scale, lab assessment, CT chest of the cases.

\begin{tabular}{|c|c|c|c|c|c|c|c|}
\hline & Case 1 & Case 2 & Case 3 & Case 4 & Case 5 & Case 6 & Case7 \\
\hline $\begin{array}{l}\text { Clinical status \& } \\
\text { performance }\end{array}$ & $\begin{array}{l}\text { WHO } \\
\text { score } 3\end{array}$ & $\begin{array}{l}\text { WHO } \\
\text { score } 1\end{array}$ & $\begin{array}{l}\text { WHO } \\
\text { score } 7\end{array}$ & WHO score 4 & $\begin{array}{l}\text { WHO assessment } \\
\text { score } 1\end{array}$ & $\begin{array}{l}\text { WHO } \\
\text { score } 3\end{array}$ & WHO score 2 \\
\hline WBC & 10 & 12 & 12.5 & 8 & 5.2 & 3.9 & 5.7 \\
\hline Lymphocyte count & 0.90 & 1.2 & 0.80 & 0.71 & 1.2 & 0.98 & 2.5 \\
\hline D. Dimer & & & & 0.69 & 0.15 & & \\
\hline Creatinine & 0.9 & 1.12 & 2.5 & 1.5 & 1.08 & 0.8 & 0.9 \\
\hline Bilirubin (T) & 3.7 & 1.1 & 33 & 2.2 & 0.57 & 0.9 & 5 \\
\hline ALT/ AST(U/L) & $33 / 36$ & $45 / 55$ & $43 / 53$ & $210 / 118$ & $55 / 54$ & $15 / 7$ & $112 / 125$ \\
\hline Ferritin & 500 & 550 & 1500 & 1029 & 107 & 120 & 100 \\
\hline CRP (mg/dl) & 206 & 55 & 108 & 24 & 27.5 & 30.5 & 6.7 \\
\hline Chest CT scan & GGO (CORAD 3) & GGO (CORAD 3) & $\begin{array}{l}\text { Bilateral GGO } \\
\text { (CORAD 5) }\end{array}$ & $\begin{array}{l}\text { Bilateral GGO } \\
\text { CORAD } 5\end{array}$ & NAD & $\begin{array}{l}\text { Subpleural faint } \\
\text { opacity on one } \\
\text { side (CORAD 1) }\end{array}$ & $\begin{array}{l}\text { Sub pleural faint } \\
\text { opacity (CORAD } \\
\text { 1) }\end{array}$ \\
\hline $\begin{array}{l}\text { SARS-CoV-2 real-time } \\
\text { PCR }\end{array}$ & Positive & Positive & Negative & Positive & Positive & Positive & Negative \\
\hline Required hospitalization & Yes & No & Yes & Yes & No & Yes & No \\
\hline & Case 8 & Case 9 & Case 10 & Case 11 & Case 12 & Case 13 & Case14 \\
\hline $\begin{array}{l}\text { Clinical status \& } \\
\text { performance }\end{array}$ & $\begin{array}{l}\text { WHO } \\
\text { score } 3\end{array}$ & $\begin{array}{l}\text { WHO } \\
\text { score } 3\end{array}$ & $\begin{array}{l}\text { WHO } \\
\text { score } 3\end{array}$ & WHO score 2 & $\begin{array}{l}\text { WHO assessment } \\
\text { score } 1\end{array}$ & $\begin{array}{l}\text { WHO } \\
\text { score } 1\end{array}$ & WHO score 1 \\
\hline WBC & 2.5 & 10 & 12.5 & 7 & 8.5 & 3 & 7 \\
\hline Lymphocyte count (\%) & 0.10 & 2.5 & 0.98 & 0.75 & 2.1 & 1.9 & 2 \\
\hline D. Dimer & 1.1 & & & 2.5 & 1.5 & & 3.2 \\
\hline Creatinine & 0.5 & 1 & 1.6 & 3.5 & 0.08 & 0.7 & 1.1 \\
\hline Bilirubin (T) & 1.1 & 1.1 & 0.35 & 5.5 & 0.51 & 0.95 & 5 \\
\hline ALT/ AST(U/L) & $12 / 25$ & $42 / 15$ & $43 / 53$ & $550 / 102$ & $42 / 20$ & $11 / 20$ & $500 / 250$ \\
\hline Ferritin & 324 & 890 & 1020 & 8059 & 25 & 220 & 1000 \\
\hline CRP (mg/dl) & 142 & 155 & 86 & 240 & 20 & 30.5 & 6.7 \\
\hline Chest CT scan & GGO(CORAD 1) & GGO(CORAD 2) & $\begin{array}{l}\text { Bilateral GGO } \\
\text { (CORAD 5) }\end{array}$ & $\begin{array}{l}\text { Bilateral GGO } \\
\text { CORAD } 3\end{array}$ & NAD & NAD & $\begin{array}{l}\text { BILATERAL } \\
\text { GCO(CORAD 4) }\end{array}$ \\
\hline $\begin{array}{l}\text { SARS-CoV-2 real-time } \\
\text { PCR }\end{array}$ & Negative & Positive & Negative & Positive & Positive & Positive & Negative \\
\hline \multirow[t]{2}{*}{ Required hospitalization } & Yes & yes & Yes & $\begin{array}{l}\text { managed at } \\
\text { home }\end{array}$ & No & no & No \\
\hline & Case 15 & Case 16 & Case 17 & Case 18 & Case 19 & Case 20 & Case21 \\
\hline $\begin{array}{l}\text { Clinical status \& } \\
\text { performance }\end{array}$ & $\begin{array}{l}\text { WHO } \\
\text { score } 4\end{array}$ & $\begin{array}{l}\text { WHO } \\
\text { score } 2\end{array}$ & $\begin{array}{l}\text { WHO } \\
\text { score } 3\end{array}$ & WHO score 3 & $\begin{array}{l}\text { WHO assessment } \\
\text { score } 1\end{array}$ & $\begin{array}{l}\text { WHO } \\
\text { score } 2\end{array}$ & WHO score 2 \\
\hline WBC & 3.5 & 1.5 & 5.5 & 8 & 6.2 & 9.4 & 7.8 \\
\hline Lymphocyte count & 0.28 & 1.1 & 0.77 & 1.71 & 2.2 & 0.55 & 3.1 \\
\hline D. Dimer & & 2.5 & & & & & \\
\hline Creatinine & 1.5 & 1.1 & 1.9 & 2.4 & 0.08 & 1.5 & 1.9 \\
\hline Bilirubin (T) & 1.1 & 1.2 & 0.33 & 0.25 & 0.55 & 0.19 & 2.5 \\
\hline ALT/ AST(U/L) & $13 / 25$ & $25 / 15$ & $102 / 150$ & $117 / 278$ & $15 / 22$ & $11 / 10$ & $177 / 250$ \\
\hline Ferritin & 280 & 550 & 1000 & 2505 & 88 & 259 & 900 \\
\hline CRP (mg/dl) & 206 & 55 & 108 & 24 & 27.5 & 30.5 & 6.7 \\
\hline Chest CT scan & $\begin{array}{l}\text { GGO } \\
\text { CORAD 5) }\end{array}$ & GGO(CORAD 2) & $\begin{array}{l}\text { Bilateral GGO } \\
\text { (CORAD 5) }\end{array}$ & $\begin{array}{l}\text { Bilateral GGO } \\
\text { CORAD } 5\end{array}$ & NAD & NAD & $\begin{array}{l}\text { Sub pleural faint } \\
\text { opacity(CORAD 1) }\end{array}$ \\
\hline $\begin{array}{l}\text { SARS-CoV-2 real-time } \\
\text { PCR }\end{array}$ & Positive & Positive & POSITIVE & Positive & Positive & Positive & POSITIVE \\
\hline Required hospitalization & yes & No & Yes & Yes & No & no & No \\
\hline
\end{tabular}

Abbreviations: CRP: C-Reactive Protein (<5 mg/L); d: Day; D-dimer normal value < $500 \mathrm{ng} / \mathrm{mL}$; FK: Tacrolimus; HCQ: Hydroxy Chloro Quine sulfate; MMF: Sodium Mycophenolate; Normal reference value of lymphocytes $=1.5-3.510^{3} / \mathrm{mm}^{3}$; WBC: White Blood Cells $\left(4-1110^{3} / \mathrm{mm}^{3}\right)$.

consent, based on the severity of COVID-19 illness and the risk of rejection.

$52.1 \%$ of mild ambulatory cases with normal $\mathrm{SO}_{2}$ (WHO score 1,2) were managed at home, $47.1 \%$ required hospitalization (WHO score 3,4 ) and a single case $4.7 \%$ required ICU admission with ventilation and renal replacement therapy together with inotropes due to multi-organ failure.
Out of the $47.1 \%$ hospitalized only one required Mechanical ventilation and the other 2 cases required nasal prongs with low oxygen requirements. Steroids were required in 10 (47\%) cases due to evident of rapid lung involvement and elevated inflammatory markers.

Patients with symptoms restricted to the upper respiratory tract, normal oxygen saturation and no radiologic features 
Citation: Ismail A, Mohamed S, Mahmoud E, et al. (2021) Outcome of Corona Virus Disease (COVID-19) in Egyptian Cohort of Long-Term Liver Transplant Recipients: Single Center Experience. J Transplant Surg 4(2):80-86

Table 3: Outcome of the cases.

\begin{tabular}{|c|c|c|c|c|c|c|c|}
\hline & Case 1 & Case 2 & Case 3 & Case 4 & Case 5 & Case 6 & Case 7 \\
\hline Oxygen therapy & No & No & Yes & Yes & No & No & No \\
\hline Mechanical ventilation & No & No & Yes & No & No & No & No \\
\hline $\begin{array}{l}\text { Modification of IS } \\
\text { drugs }\end{array}$ & No & No & $\begin{array}{l}\text { Yes } \\
\text { (Reduction in tac } \\
\text { and MMF) then D.C }\end{array}$ & Yes/Reduction & No & No & No \\
\hline Received antiviral & No & No & No & No & No & No & No \\
\hline Antibiotics & AZM & $\begin{array}{l}3^{\text {rd }} \text { G. CSP } \\
+ \text { AZM }\end{array}$ & $\begin{array}{l}\text { Imipenem } \\
\text { Cilastatin } \\
\text { AZM }\end{array}$ & $\begin{array}{l}3^{\text {rd }} \text { G. CSP } \\
+ \text { AZM }\end{array}$ & No & AZM & No \\
\hline HCQ & Yes & Yes & No & Yes & No & No & No \\
\hline Steroids or biological & No & No & $\begin{array}{l}\text { Yes } \\
\text { IV steroids } \\
100 \mathrm{mg}\end{array}$ & $\begin{array}{l}\text { Oral Steroids/ } \\
\text { Antifungal }\end{array}$ & No & No & No \\
\hline C. Plasma & No & No & No & No & No & No & No \\
\hline \multirow[t]{2}{*}{ Outcomes } & Improved & Improved & Died & Improved & Improved & Improved & Improved \\
\hline & Case 8 & Case 9 & Case 10 & Case 11 & Case 12 & Case 13 & Case 14 \\
\hline Oxygen therapy & no & no & no & no & No & No & No \\
\hline Mechanical ventilation & No & No & no & No & No & No & No \\
\hline $\begin{array}{l}\text { Modification of IS } \\
\text { drugs }\end{array}$ & No & No & no & Yes/Reduction & No & No & No \\
\hline Received antiviral & No & No & No & No & No & No & No \\
\hline Antibiotics & AZM & $\begin{array}{l}3^{\text {rd }} \text { G. CSP } \\
+ \text { AZM }\end{array}$ & Imipenem & $\begin{array}{l}3^{\text {rd }} \text { G. CSP } \\
+ \text { AZM }\end{array}$ & No & AZM & No \\
\hline HCQ & NO & NO & No & NO & No & No & No \\
\hline Steroids or biological & YES & No & Yes & Oral Steroids & No & No & yes \\
\hline C. Plasma & No & No & No & No & No & No & No \\
\hline \multirow[t]{2}{*}{ Outcomes } & Improved & Improved & IMPROVED & Improved & Improved & Improved & Improved \\
\hline & Case 15 & Case 16 & Case 17 & Case 18 & Case 19 & Case 20 & Case 21 \\
\hline Oxygen therapy & yes & No & no & no & no & No & no \\
\hline $\begin{array}{l}\text { Mechanical } \\
\text { ventilation }\end{array}$ & No & No & no & No & No & No & No \\
\hline $\begin{array}{l}\text { Modification of IS } \\
\text { drugs }\end{array}$ & yes & No & no & no & No & No & No \\
\hline Received antiviral & No & No & No & No & No & No & No \\
\hline Antibiotics & $\begin{array}{l}\text { AZM } \\
\text { Carbapenems }\end{array}$ & $3^{\text {rd }}$ G. CSP & AZM & $3^{\text {rd }}$ G. CSP & No & no & No \\
\hline HCQ & no & no & No & no & No & No & No \\
\hline Steroids or biological & yes & yes & $\begin{array}{l}\text { Yes } \\
\text { Oral steroids }\end{array}$ & Iv Steroids & No & yes & No \\
\hline C. Plasma & No & No & No & No & No & No & No \\
\hline Outcomes & Improved & Improved & improved & Improved & Improved & Improved & Improved \\
\hline
\end{tabular}

of pneumonia have been treated at home with isolation measures [12,13]. Carbapenems were added 2 of to our cases showing respiratory failure and increasing inflammatory parameters together with intravenous corticosteroids. Antifungal therapy was added to a single case not responding to conventional antibiotics with aggressive lung involvement with good clinical response.

Only 1 case (4.7\%) died due to multi-organ failure and the rest $92 \%$ shows clinical improvement without any long term oxygen requirements.

\section{Adjustment of immunosuppressive regimen}

All transplant patients received routine immunesuppressive treatment (Table 1). According to our immunosuppressive protocol, LT recipients receive triple immunosuppression in the first year (All patients received calcineuirne inhibitors, mainly Tacrolimus with or without
Mycopenolate (MMF) or m-TOR inhibitors) [14]. The management of immunosuppression was left according to clinical severity and judgment, Two of the patients required cessation of all immunosuppressive drugs, other two required reducing doses by $50 \%$ and the rest were kept on the same doses of immunosuppressive drugs, although tapering of maintenance therapy was usually attempted in only one case which showed symptoms of respiratory failure and worsening of chest findings with tapering the Tacrolimous dose, however the rest of the cases no dose adjustment of immunosuppressive was done and all patient were followed via telemedicine during their home isolation period.

All patients had elevated Ferritin, which is common in COVID-19, related to severity and mortality, and this finding reinforces the recommendations of American Association for Study of Liver Diseases (AASLD) to withdrawal antimetabolite during hospitalization for COVID-19 [15]. 
Citation: Ismail A, Mohamed S, Mahmoud E, et al. (2021) Outcome of Corona Virus Disease (COVID-19) in Egyptian Cohort of Long-Term Liver Transplant Recipients: Single Center Experience. J Transplant Surg 4(2):80-86

Table 4: Summarizes most important study points.

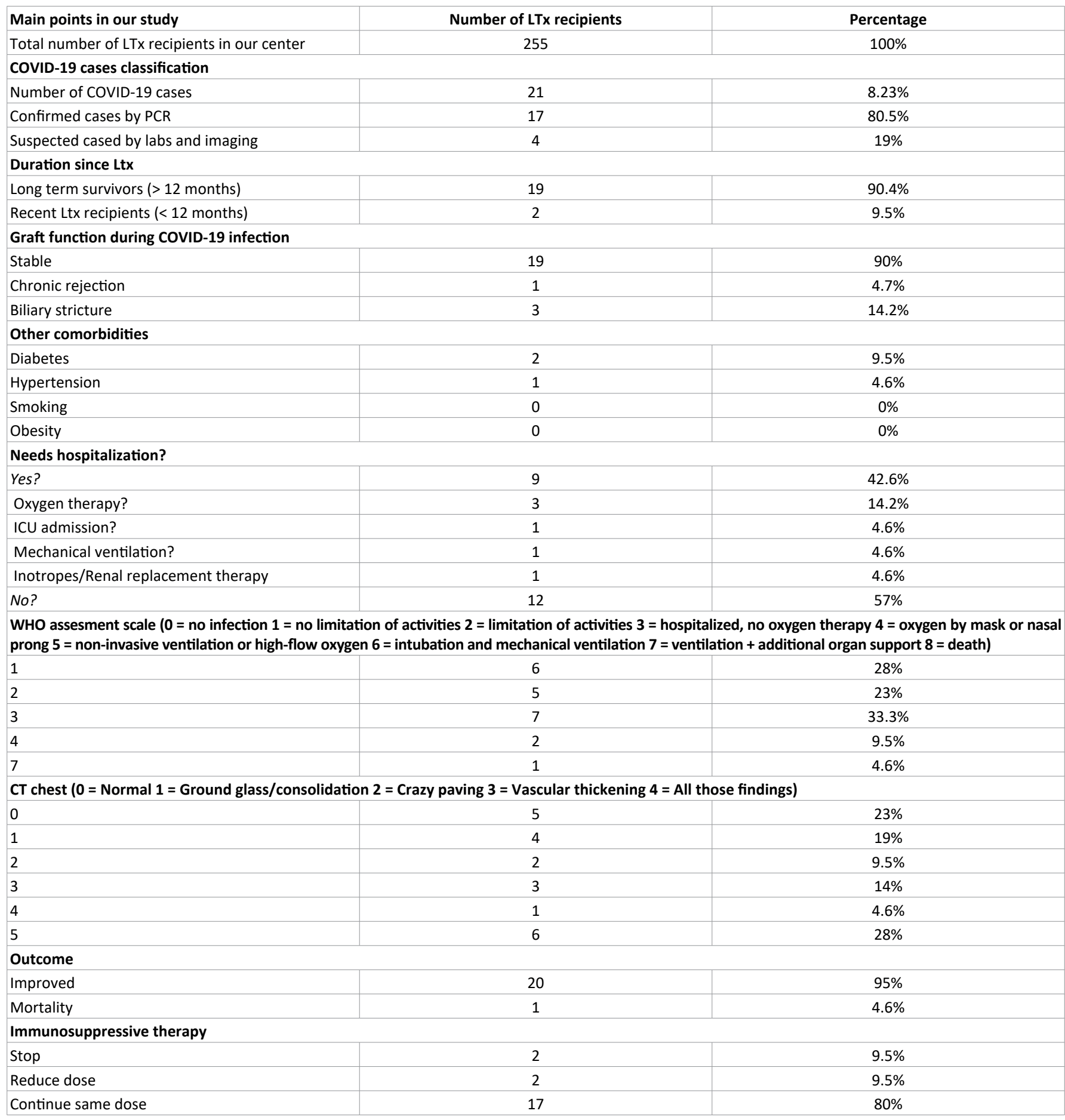

It appears that immunosuppression doesn't seem to have protective effect neither a harmful one as well and age and other co-morbidities together with biliary complications could worsen the prognosis of disease, such as in general population. It is important to closely monitor these patients [16].

\section{Discussion}

The World Health Organization (WHO) declared COVID-19 as a pandemic on March 11, 2020 [17]. In this article we tried to report our experience with seven cases of wide spectrum of clinical picture from mild up to severe requiring mechanical ventilation, we also tried to review and summarize literature about further management of liver transplantation cases during COVID-19 pandemic.

In viewing our cases and those reported in the literature, we noticed that the symptoms, laboratory values, and imaging in $\mathrm{LT}$ recipients were similar to those of immune competent patients without clear difference between the two categories [18].

There were some limitations in our study. First, this is a single-center analysis of a limited number of patients. Second, there is a limited duration of the follow up for the infected cases, as it has been in many clinical COVID-19 studies given the need to rapidly share knowledge in this quickly evolving 
Citation: Ismail A, Mohamed S, Mahmoud E, et al. (2021) Outcome of Corona Virus Disease (COVID-19) in Egyptian Cohort of Long-Term Liver Transplant Recipients: Single Center Experience. J Transplant Surg 4(2):80-86

pandemic. Last and most important, the role of serologic testing and immunity checkup as a diagnostic tool in organ transplantation patients is still not clear. There is a risk of false positives, particularly with IgM testing, and a lack of clear evidence on which antibodies are surrogates of protection or potent at neutralizing virus [19].

\section{Conclusion}

We report our experience with 21 Solid organ liver transplantation patients with COVID-19 and found that, Male gender, advanced age, Comorbidities and Mycophenolate were associated with SARS-CoV-19 mortality in liver transplant recipients. Modification of immunosuppressive drugs is not mandatory in most of cases except for MMF which should be discontinued. Although incidence of SARS-COV-19 could be higher in LTx recipients, but mortality could be same or even lower than General population due to possible protective effect of chronic immunosuppression despite immune suppression. Notably, One liver transplant recipient at our institution have died despite existing literature documenting increased mortality in this population, emphasizing the importance of further studies to determine which organ transplantation subgroups who may have more favorable outcomes and which who may be at great risk for cytokine storm and further multiorgan failure. Additional research is urgently needed to close the knowledge gap regarding COVID-19 among liver transplantation recipients.

\section{Conflict of Interest}

The authors who have taken part in this study declared that they did not have anything to disclose regarding funding or conflict of interest with respect to this manuscript.

\section{Authors Contribution}

Mahmoud Essam El-Din: Paper writing and data collection; Ismail Anwar: Data collection and corresponding author; Mohamed Said: Revision of the article; Mostafa El Shazly: Revision of the article; Karim Hosny: Revision of the article; Naglaa Zayed: Revision of the article.

\section{References}

1. Michaels MG, La Hoz RM, Danziger-Isakov L, et al. (2020) Coronavirus disease 2019: Implications of emerging infections for transplantation. Am J Transplant 20: 1768-1772.

2. Zhu NA, Zhang D, Wang W, et al. (2020) A novel coronavirus from patients with pneumonia in China, 2019. N Engl J Med 382: 727-733.

3. Zhu L, Xu X, Ma K, et al. (2020) Successful recovery of COVID-19 pneumonia in a renal transplant recipient with long-term immunosuppression. Am J Transplant 20: 1859-1863.
4. Guillen E, Pineiro GJ, Revuelta I, et al. (2020) Case report of COVID-19 in a kidney transplant recipient: Does immunosuppression alter the clinical presentation? Am J Transplant 20: 1875-1878.

5. Cordero E, Perez-Rosmero P, Moreno A, et al. (2012) Pandemic influenza $A(H 1 N 1)$ virus infection in solid organ transplant recipients: Impact of viral and non-viral co-infection. Clin Microbiol Infect 18: 67-73.

6. Mehta P, McAuley DF, Brown M, et al. (2020) COVID-19: Consider cytokine storm syndromes and immunosuppression. Lancet 395: 1033-1034.

7. Lee BT, Perumal swami PV, Im GY, et al. (2020) COVID-19 in liver transplant recipients: An initial experience from the US epicenter. Gastroenterology 159: 1176-1178.

8. National Health Commission of the People's Republic of China (2020).

9. Centers for Disease Control and Prevention (CDC) (2019) RealTime RT PCR Panel for Detection 2019-Novel Coronavirus. Centers for Disease Control and Prevention, Atlanta, USA.

10. WHO (2020) COVID-19 assessment scale.

11. Zhou S, Wang Y, Zhu T, et al. (2020) CT features of coronavirus disease 2019 (COVID-19) pneumonia in 62 patients in Wuhan, China. AJR Am J Roentgenol 214: 1287-1294.

12. Tobaiqy M, Qashqary M, Al-Dahery S, et al. (2020) Therapeutic management of COVID-19 Patients: A systematic review. Infection Prevention in Practice 2: 100061.

13. Sorour K, El-Menshawy H (2020) A proposed protocol for the management of COVID-19 in Egypt.

14. Burra P, Burroughs A, Graziadei I, et al. (2016) EASL clinical practice guidelines: Liver transplantation. J Hepatol 64: 433485.

15. Fix OK, Hameed B, Fontana RJ, et al. (2020) Clinical best practice advice for hepatology and liver transplant providers during the COVID-19 pandemic: AASLD expert panel consensus statement. Hepatol 72: 287-304.

16. Fernández-Ruiz M, Andrés A, Loinaz C, et al. (2020) COVID-19 in solid organ transplant recipients: A single-center case series from Spain. Am J Transplant 20: 1849-1858.

17. World Health Organization (2020) Coronavirus disease 2019 (COVID-19).

18. Zhong Z, Zhang $\mathrm{Q}$, Xia $\mathrm{H}$, et al. (2020) Clinical characteristics and immunosuppressants management of coronavirus disease 2019 in solid organ transplant recipients. Am J Transplant 20: 1916-1921.

19. Fung M, Chiu CY, DeVoe C, et al. (2020) Clinical outcomes and serologic response in solid organ transplant recipients with COVID-19: A case series from the United States. Am J Transplant 20: 3225-3233.

DOI: $10.36959 / 338 / 338$

Copyright: (c) 2021 Ismail A, et al. This is an open-access article distributed under the terms of the Creative Commons Attribution License, which permits unrestricted use, distribution, and reproduction in any medium, provided the original author and source are credited. 